\title{
GROUP A HEMOLYTIC STREPTOCOCCUS ANTIBODIES. II. GRIFFITH TYPE AGGLUTININ AND ANTISTREPTOLYSIN TITERS IN CARRIERS AND NON-CARRIERS
}

\author{
By LOWELl A. RANTZ, ALVIN H. JACOBS, AND WILlIAM M. M. KIRBY \\ (From the Departments of Medicine and Pediatrics, Stanford University \\ School of Medicine, San Francisco)
}

(Received for publication November 5, 1942)

In the previous paper, hemolytic streptococcus antibacterial and antitoxic antibodies were studied by the determination of agglutinins for various Griffith types and antistreptolysin, in the sera of normal individuals who were not shown to be nasopharyngeal carriers of Group A streptococci. It is the purpose of this report to describe a more extensive study of these antibodies in carriers and non-carriers of hemolytic streptococci of Group A. This program was prompted by two previous observations.

First, and most important, were the carefully controlled studies of Bloomfield and Felty (1) who, in 1926, pointed out that individuals harboring hemolytic streptococci in the tonsils were immune to infection by these organisms until the carrier state had terminated. It therefore seemed possible that high titers of circulating antibody might be demonstrable in the sera of Group A carriers.

A further incentive to this work arose from the study of circulating antibodies in individuals suffering acute attacks of hemolytic streptococcus pharyngitis $(2,3)$, in whom it was observed that very great variations existed from patient to patient in the level of antistreptolysin at the onset of the infection and in the rate of fall after recovery. The high titers demonstrated in some of these individuals might have been maintained by a prolongation of the carrier state after previous infection. The study of hemolytic streptococcus carriers is, however, greatly complicated by the fact, previously emphasized (4), that ordinary throat swabs will recover the organism in a relatively small number of cases, since it inhabits the crypts and not the surface of the tonsil. This is particularly true if no recent episode of acute infection has occurred.

In the present study, sera for antibody determinations were obtained from children who were about to undergo tonsillectomy and the entire excised tonsil was then examined by cultural methods suitable for the isolation of Group A streptococci. This procedure has permitted the precise separation of these individuals into carriers and non-carriers.

The results of the determination of the levels of circulating antibodies in this group will now be presented.

\section{MATERIALS AND METHODS}

Sera, throat swabs, and a careful history were obtained from 64 children who were about to undergo tonsillectomy. Later, the excised tonsils were cultured.

\section{ANTIBODY DETERMINATION}

The levels of circulating agglutinins for the various common local types of Group A streptococci and of antistreptolysin were determined in the same manner as that described in the previous report (2).

\section{BACTERIOLOGICAL OBSERVATIONS}

Throat swabs and excised tonsils were studied culturally and the isolated hemolytic streptococci were classified serologically by means of the techniques that have been described in detail elsewhere $(4,5)$. Group $A$ hemolytic streptococci were recovered from preoperative throat swabs in only 55 per cent of individuals, later proved to be carriers by culture of the excised tonsils.

\section{RESULTS}

Of the 64 children studied, 33 were proved to be carriers of Group A hemolytic streptococci.

Because the proximity of active infection is important in the consideration of antibody mechanics, a history of recent or remote streptococcus infection was sought in each case. Thirty per cent of the carriers and 37.1 per cent of the noncarriers had suffered a sore throat within 4 weeks, and 66.6 per cent and $\mathbf{5 1 . 9}$ per cent, respectively, within 16 weeks. Two in each group had had scarlet fever. No correlation between a history 
of recent infection and the level of circulating antibodies could be established.

Agglutinins and antistreptolysin. In Figure 1, the results of the determination of agglutinins and antistreptolysin, in relation to the presence or absence of the carrier state, are correlated graphically. Agglutinins were determined, using antigens prepared from Group A streptococci of Types $1,2,4,6,9,11,12,13$, and 25 , and also, in carriers, the homologous type if it was not included in the previous group. In the figure, the highest agglutinin titer for any type was plotted. In 27 per cent of the carriers, this was not the homologous type. These cross reactions have been described in the previous paper.

Of group A carriers, 84.9 per cent had agglutinin titers greater than $1: 8$, and in only 9.1 per cent, were no agglutinins demonstrated. Among the non-carriers, the situation was entirely different. Here, titers above 1:8 were present in only 12.8 per cent of the group, and 61.5 per cent had no agglutinins for any of the studied types.

Somewhat similar but less dramatic were the results obtained by correlating antistreptolysin titers with the presence or absence of the carrier state. The figure reveals that 45.5 per cent of individuals who harbored Group A streptococci in the nasopharynx had antistreptolysin titers above
250 units and only 30.2 per cent were as low as 100 units or less. The level of antistreptolysin was above 250 units in 22.4 per cent, and 100 units or less in 61.5 per cent of the non-carriers. It is of interest to note that Group C hemolytic streptococci were isolated from the tonsils of 2 , and Group G from 1 of the 7 individuals in this group whose antistreptolysin titers were greater than 250 units.

Follow-up studies. When it became apparent that elevated antibody titers were demonstrable in most of the carriers of Group A streptococci, it became desirable to determine the fate of these substances after termination of the carrier state by tonsillectomy. Consequently, 21 of the Group A carriers were restudied after intervals from 26 to 100 days, and the levels of agglutinins and antistreptolysin were determined. Throat cultures were obtained and in no case were Group A streptococci resident in the nasopharynx at the time of re-examination. Follow-up antistreptolysin titers are charted in Figure 2. It will be observed that definite fall in the level of this antibody occurred in 19 of the 21 individuals and that the rate and relative amount of this change was fairly constant for any initial antibody titer.

Similar results were not obtained in follow-up studies of the agglutinin titers. Satisfactory ob-

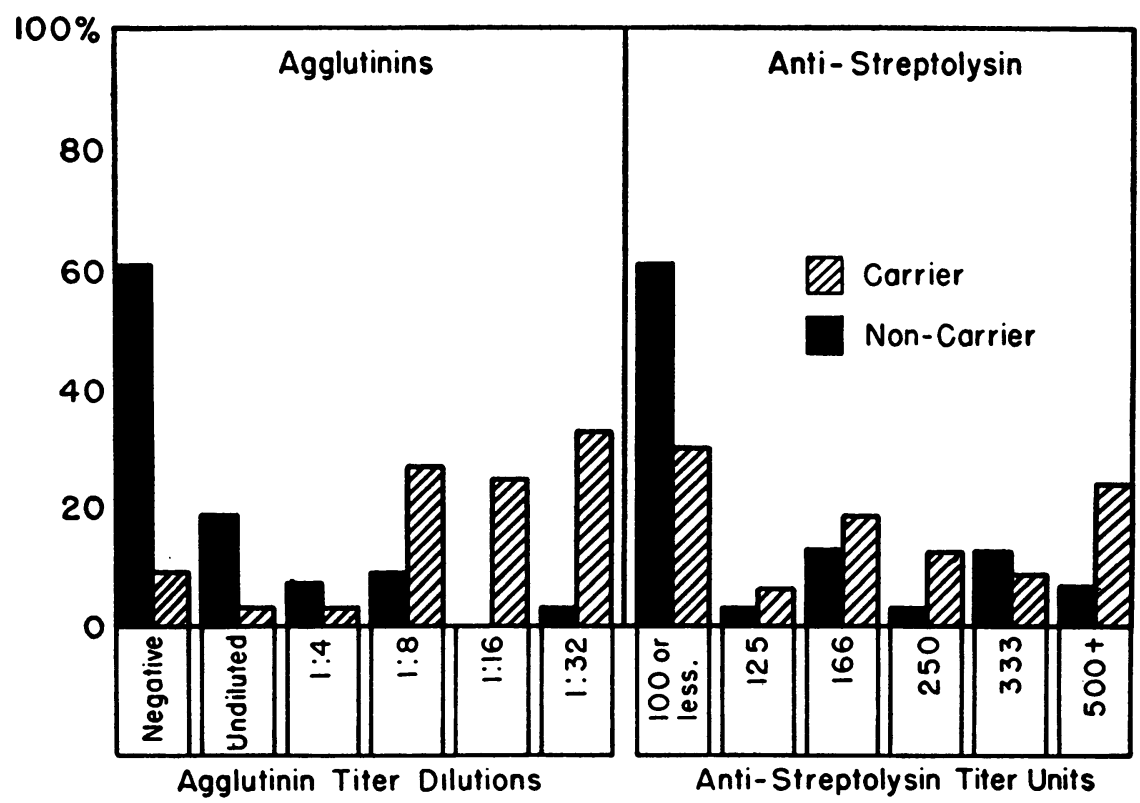

Fig. 1. Agglutinin and Antistreptolysin Titers in Carriers and Non-Carriers of Group A Hemolytic Streptococci 


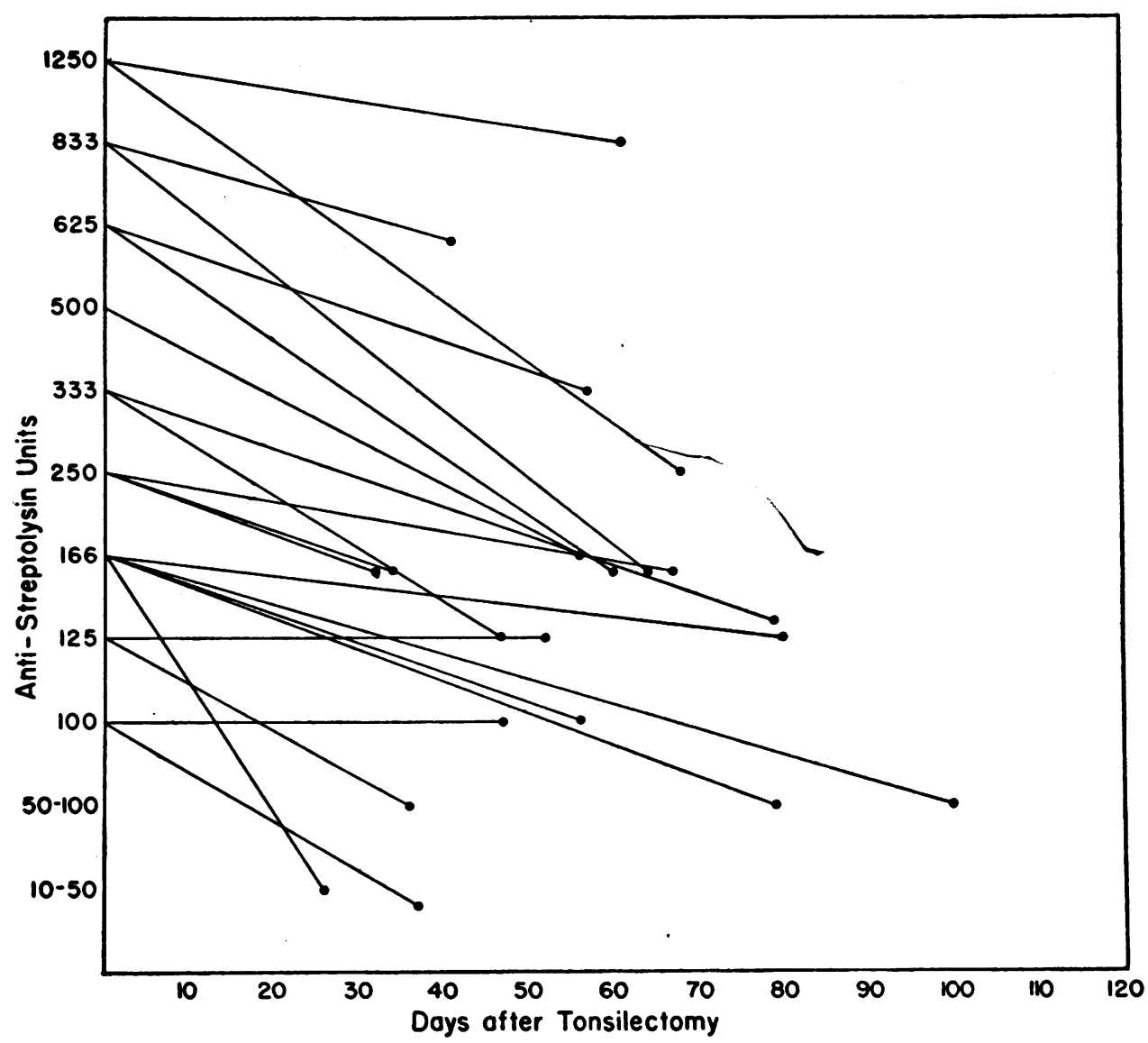

Fig. 2. Fall in Antistreptolysin Titer in 21 Group A Hemolytic Streptococcus Carriers After Tonsthlectomy

servations were available in 17 cases from 40 to 80 days after tonsillectomy. No change had occurred in the agglutinin levels of 9,5 had fallen 1 dilution, and 3 had fallen 2 dilutions. These changes were not correlated in any way with the interval of time since operation.

Results of Griffith typing. The Griffith types of the isolated Group A streptococci were determined, the distribution being presented in Table $\mathrm{I}$.

Types 6,12 , and 25 were most frequently isolated from the tonsils of these individuals. In 9

TABLE I

Distribution of Griffith types of group $A$ streptococci from excised tonsils

\begin{tabular}{c|c|c|c|c|c|c|c|c|c|c|c|c}
\hline Griffith types & 1 & 2 & 4 & 6 & 9 & 11 & 12 & 22 & 23 & 25 & 27 & $\begin{array}{c}\text { Uni- } \\
\text { denti- } \\
\text { fied }\end{array}$ \\
\hline $\begin{array}{c}\text { Number of } \\
\text { strains }\end{array}$ & 2 & 1 & 3 & 6 & 1 & 2 & 5 & 1 & 1 & 6 & 1 & 4 \\
\hline
\end{tabular}

instances, agglutinins for the homologous type were absent from the serum of carriers. Two of these children harbored type $4 ; 3$, type 6 ; and 3 , unidentified types. In 6 , agglutinins for other types were discovered. No other correlation between Griffith type and any antibody level was established.

\section{DISCUSSION}

The observations described in this paper indicate that tonsillar carriers of Group A streptococci have elevated levels of circulating antistreptococcal antibodies more frequently than do noncarriers. These differences are most easily demonstrable by the study of agglutinins, since nearly all carriers have markedly increased amounts of these antibodies and only a few noncarriers do so. A similar but less marked trend is apparent when the antistreptolysin titers are considered. 
The interpretations of these results is difficult since two obvious causes for these variations in antibody level may be advanced. First, the presence of hemolytic streptocococci in the tonsil may stimulate the maintainance of increased amounts of antibody or, second, carriers may simply have more recently undergone an active hemolytic streptococcus infection. The last hypothesis is not borne out by the histories obtained from the studied individuals or their families, but the value of such data in regard to respiratory infections in children is questionable.

A study of the antibody levels for a long time before and after tonsillectomy is necessary for the adequate evaluation of the importance of the carrier state in their maintenance. The follow-up observations in this study after operation are of interest, however, because of the consistent fall in antistreptolysin titer over a two-month period. A careful consideration of previous studies indicates that no such constant relationship exists if the antistreptolysin titers are followed over long periods, after an acute infection. While there is always a general tendency for the titers to return to normal with the passage of time, yet antistreptolysin titers greater than 400 units were present 6 months after an acute streptococcal respiratory infection in 49 per cent of the group studied by Mote and Jones (3). These same authors followed 12 cases at frequent intervals after an acute infection. Usually the antistreptolysin titer was well maintained for a long time or fell very slowly. In about half, the titers decreased rapidly after remaining at a high level for several months.

In the present group, the antistreptolysin titers fell markedly in 90 per cent of the cases during an interval of about 2 months. It seems reasonable to suppose that the stimulus to this constant occurrence, so unlike that observed by others, was the termination of the carrier state by tonsillectomy, and that the presence of Group A streptococci in the tonsil did contribute to the maintenance of increased amounts of antistreptolysin.

The high antistreptolysin titers discovered in a small number of non-carriers may be explained in three ways. Half of this group harbored strains of Groups $C$ and $G$ in the tonsil, and it has been shown elsewhere (6) that certain members of these groups are toxigenic and produce strep- tolysin, antigenically similar to that of Group A. The presence of these organisms may have stimulated and maintained high levels of antistreptolysin. It is possible that in the others, a previous Group A carrier state was terminated spontaneously just before the individuals came under study, or that the cultural techniques failed in an occasional instance to recover the hemolytic streptococci resident in the tonsil.

A consistent fall of agglutinin titer after tonsillectomy was not observed and much more detailed study will be necessary to determine the importance of the carrier state for the maintainance of increased amounts of these antibodies, but it is possible that titers above $1: 8$ are stimulated by hemolytic streptococci in the nasopharynx, since these levels are rarely found in the sera of noncarriers.

The presence of large amounts of circulating antibacterial and antitoxic antibodies in the sera of Group A hemolytic streptococcus carriers cannot be interpreted as evidence that such individuals are immune to infection by these organisms, since the protective value of these substances is in no sense established. It is of great interest, however, that these evidences of potential immunity should be so constantly present in a group of individuals that has been previously demonstrated, by Bloomfield and Felty, to be extremely resistant to infection. Further studies for the purpose of clarifying these important points are in progress and will be described later.

\section{SUMMARY}

1. The Group A hemolytic streptococcus agglutinin and the antistreptolysin titers of the sera of 64 children were measured just previous to tonsillectomy.

2. Thirty-three of these individuals were demonstrated to be Group A carriers by culture of the excised tonsils.

3. The sera of carriers contained larger amounts of agglutinins and antistreptolysin than did that of non-carriers.

4. When the carrier state was terminated by tonsillectomy, there was a constant decline in antistreptolysin titer within 60 days, and a less regular fall in agglutinin titer.

5. It seems probable that the presence of Group A streptococci in the tonsil contributes to the 
maintainance of elevated levels of antistreptolysin and possibly of agglutinins.

\section{BIBLIOGRAPHY}

1. Bloomfield, A. L., and Felty, A. R., Bacteriological observations on acute tonsillitis with reference to epidemiology and susceptibility. Arch. Int. Med., 1923, 32, 483.

2. Rantz, L. A., Kirby, W. M. M., and Jacobs, A. H., Group A hemolytic streptococcus antibodies. I. Type specific agglutinin and antistreptolysin titers in normal men and in acute infections. J. Clin. Invest., 1943, 22, 411.

3. Mote, J. R., and Jones, T. D., Studies of hemolytic streptococcal antibodies in control groups, rheumatic fever and rheumatoid arthritis. I, II, and III. J. Immunol., 1941, 41, 35.

4. Rantz, L. A., The hemolytic streptococci: Studies on the carrier state in the San Francisco area with notes on the methods of isolation and serological classification of these organisms. J. Infect. Dis., 1941, 69, 248.

5. Rantz, L. A., The serological classification of hemolytic streptococci of the Lancefield group A. J. Clin. Invest., 1942, 21, 217.

6. Kirby, W. M. M., and Rantz, L. A., Lancefield group C hemolytic streptococcus bacteremia cured with sulfadiazine. Report of a case with review of the literature and presentation of immunological data. Arch. Int. Med. (In press.) 